\title{
Pipeline Monitoring using Ultrasonic Sensors
}

\author{
K. Sudhamayee
}

\begin{abstract}
Pipelines used to transport oil and gas deteriorate with time due to various reasons including corrosion, deformation, cracking etc., So instead of critical-event based inspections, a means of continuous monitoring of pipelines to analyze its response as a function of time is required. Using real time data, this monitoring can be used as a means of prior warning of any unsafe condition of many types of nondestructive testing (NDT) techniques used for defect detection in pipelines, Ultrasonic testing (UT) is preferred because of its high accuracy, reliability, affordability and its capability of generating instant test report. UT works on the principle of pulse-echo method. As the received echo signal by transducer is noisy, it is generally processed using wavelet transforms and/or FFT. Later the signal is given to Artificial Neural Network (ANN) to distinguish signal with Non-Defect (ND) and signal with Defect $(D)$.

In this proposal, UT technique is used in pipeline monitoring during machining and real time work. Two calibration standards such as straight beam testing and angle beam testing are used to detect parallel defects and weld inspection respectively.
\end{abstract}

Keywords- Pipeline Monitoring; NDT; Ultrasonic Testing; Pulse Echo Method; PIG

\section{INTRODUCTION}

Pipelines are widely used in transporting large quantities of oil and gas products over long distances due to their safety, efficiency and low cost. These pipelines play an important role in modern societies and are crucial in providing needed fuels for sustaining vital functions like power generation, heating supply and transportation. However, Pipelines are subject to deterioration and degradation. As time progresses, they may suffer from different types of defects such as corrosion, deformation, cracking, etc. These defects, if not properly managed, may result in pipeline failures resulting in leaking or spilling oil or gas content to the environment, which could lead to very expensive downtime and environment hazards. Hence, different techniques of Non -destructive testing (NDT) are required to check Pipeline Integrity for reliable operations. Defect detection and identification, defect growth prediction are major steps involved in pipeline integrity.

Revised Manuscript Received on 14 August, 2019.

K.Raghavendra, Asst. Professor, Dept. of ECE, Malla Reddy Engineering College for Women(Autonomous), Secunderabad-TS Email: raghu.siiet@gmail.com ${ }^{1}$,

Swetha, Asst. Professor, Sri Indu Institute of Engineering \& Technology, Ranga Reddy-TS

swetha.viit@gmail.com

S. Naresh, Asst. Professor, Sri Indu Institute of Engineering \& Technology, Ranga Reddy-TS, surabunaresh2012@gmai.com

\section{NONDESTRUCTIVE TESTING}

Nondestructive testing (NDT) is the process of inspecting, testing and evaluating materials for discontinuities and defects without destroying the serviceability of the part of the material. In other words, when the inspection or test is completed the part can still be used. For reliable operations, different techniques of Non -destructive testing (NDT) are proposed to check material integrity, for defect detection and identification and defect growth prediction. The two main categories of NDT inspection technologies are Onsite inspection (OSI) and Inline inspection (ILI).

Though Onsite inspection technologies are comparatively inexpensive, they can determine the defects only at surface and shallow subsurface level i.e., they can detect only external defects. Where Liquid penetrate inspection (LPI) can determine surface defects with width above $0.01 \mathrm{~mm}$ and depth between $0.03 \mathrm{~mm}$ to $0.04 \mathrm{~mm}$, Magnetic particle testing (MPT) can detect defects only in ferromagnetic material, where defects are not parallel to the flux lines. Another popular onsite technique, Industrial Radiography cannot determine the size of the defect accurately. Besides, scattering and absorption of radiation by the medium affects accuracy.

ILI techniques can detect and determine the attributes of both internal and external defects of materials. ILI tools/sensors are designed to travel inside the pipelines and evaluate the pipe to collect data as they move. These tools are sophisticated electronic devices called "Smart Pipeline Inspection Gauges (PIGs)" that use Non destructive techniques to detect and size internal damage. They measure and record the defects in pipelines such as corrosion, cracks or other irregularities.3-Axis high-resolution Magnetic flux leakage (MFL), ultrasonic testing (UT), Electromagnetic acoustic transducer (EMAT), eddy current inspection (EDI) are four major techniques of NDT. Ultrasonic testing (UT) is popular because it is highly accurate, inexpensive and has higher sensitivity compared with MFL tools.

\section{ULTRASONIC TESTING}

Ultrasonic testing (UT) is one among different NDT techniques, based on the propagation of sound waves at a frequency that is beyond human hearing range, in the object or material under test, to conduct examinations and identify discontinuities in materials that are both on and below the surface of the material. 
It can be used for defect detection/evaluation, dimensional measurements, material characterization, and more.

Sound waves are mechanical vibrations that pass through a medium such as liquid, solid or gas, at a particular velocity and expected direction. When these waves bump into a boundary having a different medium, they are transmitted back. This is the principle behind ultrasonic defect detection. In solids, sound waves can be present in different modes of propagation that are characterized by the type of motion involved. The common modes of sound waves seen in UT are:

Longitudinal waves (LW): These are also called as compression waves. The LW pushes the molecules of the tested material in the same direction as the movement of the wave. This mode is generally seen in straight beam testing. Shear wave (SW): Shear waves are also known as transverse waves, propagate more slowly and at shorter wavelengths than LWs at equal frequencies and require a fluid of higher viscosity. Here, the particle motion is at right angles to the movement of the wave. These waves are seen in angle-beam testing.

\section{TRANSDUCERS}

Ultrasonic transducers, with a typical active element such as piezo-electric crystal, can transform electrical energy into sound energy and vice versa. Straight beam transducers and angle beam transducers are generally employed for defect detection.

\section{Straight Beam Transducer}

Straight beam/wave testing uses a sound beam that is introduced at 90 degrees to the surface. It is generally used to find defects that are parallel to the surface of the test material as well as discontinuities within the body of the material, such as voids or areas of porosity. The velocity of travel within the tested material must be known in order to calculate both the presence and the depth of the defect.

When the angle of incidence is 90 degrees, longitudinal waves are produced. In a flawless test piece, if the metal has full thickness equal to $\mathrm{D}$, then the distance travelled by the beam down and back will be equal to $D$. Fig. 1 shows the scheme of straight beam inspection.

Waves generated from probes S1 and S2 encounter no defects. Thus, the transit time $\mathrm{T}$ represents the sonic waves propagating from probes $\mathrm{S} 1$ and $\mathrm{S} 2$ in both cases and the reflection from the back wall of the piece, so for the full thickness the total travel will be $2 D$.

$\mathrm{V}_{\mathrm{L}}$ is the velocity of LWs within the type of metal tested, as shown in Equation (1). Let the wave propagating from probe $\mathrm{S} 3$ encountered a defect in its path. The distance from the front surface to the interface with the defect is termed as DD (Depth of Defect). Then the transit time $\mathrm{T}_{\mathrm{D}}$ would represent the sonic waves propagating from probe $\mathrm{S} 3$ as shown in equation (2) and the reflection from the interface of the defect, so the distance traveled will be 2DD.Thus, the velocity of $\mathrm{LWs} \mathrm{V}_{\mathrm{L}}$ in this case is used to determine DD.

$$
\begin{aligned}
& \mathrm{V}_{\mathrm{L}}=2 \mathrm{D} / \mathrm{T} \\
& \mathrm{V}_{\mathrm{L}}=2 \mathrm{DD} / \mathrm{T}_{\mathrm{D}}
\end{aligned}
$$

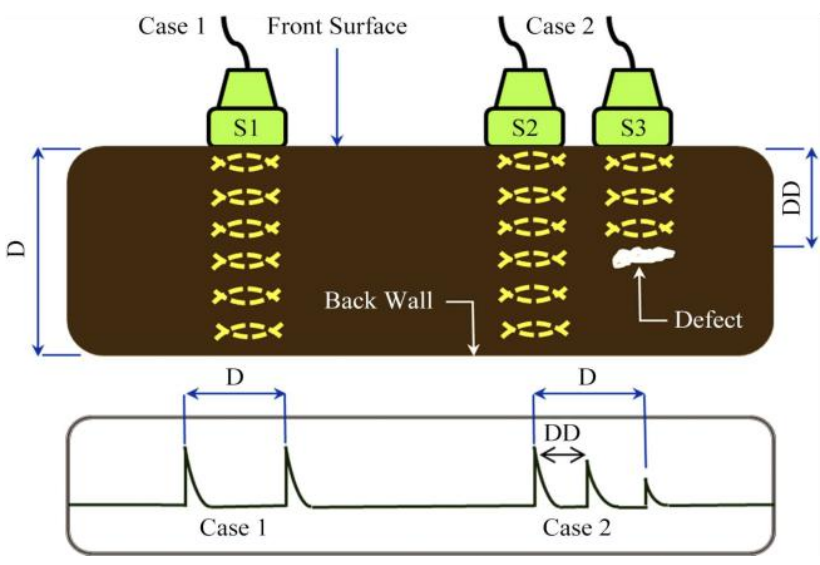

Fig.1. Scheme of Straight beam inspection

Straight beam technique can be used to determine the wall thickness. However, this technique becomes ineffective for testing welds since the discontinuities in welds are usually at an angle to the surface.

\section{Angle Beam Transducer}

If the defect is vertical and thin enough, straight beam technique does not reflect enough sound back to the transducer to let the tester know that it exists. In cases like this, angle beam ultrasound testing is used. Angle beam-testing uses an incidence angle other than 90 degrees. In contact testing, an angled plastic block is place between the transducer and the object to create the desired angle. For angle beam testing in immersion systems, a plastic block is not needed because the transducer can simply be angled in the water. If the angle of incidence is changed to be anything other than 90 degrees, longitudinal waves and shear waves are produced.

To assess discontinuities using angle beam examination, skip distance (SKD) is used to describe the sound path (SP) reflected from the back wall interface (1st leg) and going immediately to reflecting, where it is again reflected from the front wall (2nd leg). SKD is formed by the full SP (1st leg and 2nd leg), and is the distance from the point of excitation to the end of the second leg, as shown in Fig 2.

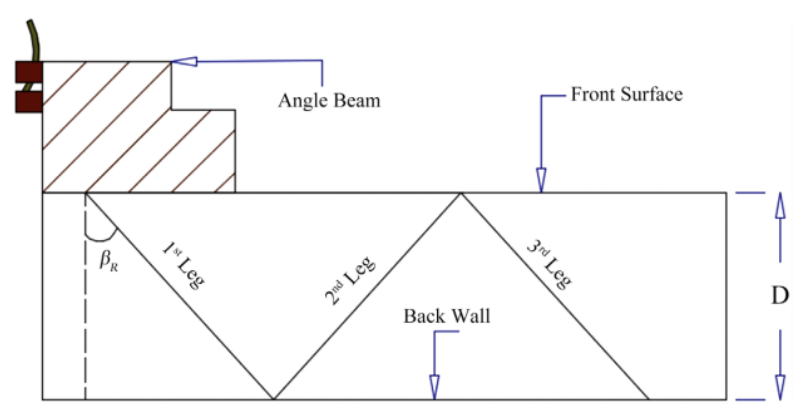

Fig.2. Representation of angle beam testing 
$\mathrm{D}$ is the thickness of the wall and $\beta_{R}$ is SP angle

$$
\mathrm{SKD}=2 D \operatorname{Tan}\left(\beta_{R}\right)
$$

Surface distance (SD) is equal to half SKD.

$\mathrm{SD}=D \operatorname{Tan}\left(\beta_{R}\right)$

These calculations cannot be completed unless $D$ and $\beta_{R}$ to the front surface are known. If the length of the 1 st leg is known, the SKD and SD can be computed as

$$
\operatorname{SKD}=2(L 1) \operatorname{Sin}\left(\beta_{R}\right)
$$

and

$$
\mathrm{SD}=L 1 \operatorname{Sin}\left(\beta_{R}\right)
$$

The segments of the SP numbered $L 1, L 2$ and $L 3$ in Fig 3 are 1 st leg $(L 1)$, 2nd leg (L2) and 3rd leg (L3) respectively. And through the trigonometric functions $L 1, L 2$ can be calculated as

$$
L 1=\mathrm{D} / \operatorname{Cos}\left(\beta_{R}\right)
$$

$L 1+L 2=2 \mathrm{D} / \operatorname{Cos}\left(\beta_{R}\right)$

To calculate the depth of the discontinuity (DD), we measure vertically from the point of reflection at the interface with the defect, up to the front surface of the test sample. Because the wave entered at an angle, it does not travel directly through the material rather it is refracted. The amount of refraction depends on the speed of sound in the two mediums between which the wave is traveling. Since the speed of shear waves is slower than the speed of longitudinal waves, their angles of refraction will be different. Hence an angle beam test cannot be performed unless the angle of refraction is calculated using snell's law.

\section{PIPELINE MONITORING USING ULTRASONIC TESTING}

UT tools/sensors are designed to travel inside the pipelines and evaluate the pipe to collect data as they move. These tools use Non destructive techniques to detect and size internal damage. UT inspection in pipelines generally follows the principle of ultrasonic measurement is based on "pulse echo time" technology. Compared with other tools, UT gives reliable defect depth sizing and good repeatability, and it can deal with very small pipeline wall thickness. UT tools require liquid coupling between the test surface and the transducer. The liquid medium carried inside the pipes provides coupling in case of oil pipes, where as a couplant is required for acoustic impedance matching in gas pipes. The couplant displaces the air and makes it possible to get more sound energy into the pipeline so that a usable ultrasonic signal can be obtained.

A typical UT inspection tool consists of a pulser/receiver, transducer, and display devices. A pulser/receiver produces high voltage electrical pulses. Driven by the pulser, the transducer generates high frequency and short wavelength ultrasonic pulses. This sound energy is introduced and propagated through the material in the form of waves. At the place where discontinuities are encountered, reduction in energy intensity and reflection/echoing of the waves by the back wall of the material is seen. The reflected/echoed signal is transformed into an electrical signal by the transducer. This signal is captured, mathematically analyzed and displayed on a screen. The display shows the reflected signal strength versus the time from signal generation to when a echo was received. Thus the resultant waveform gives the location, size, orientation and other features of defects on or within the material. The working principle of an ultrasonic sensor is shown in Fig 3. Because the sensing mode of ultrasonic evaluation is a mechanical process, the central frequency ranges from $0.1 \mathrm{MHz}$ to $25 \mathrm{MHz}$, to avoid permanent damage to the targeted objects.
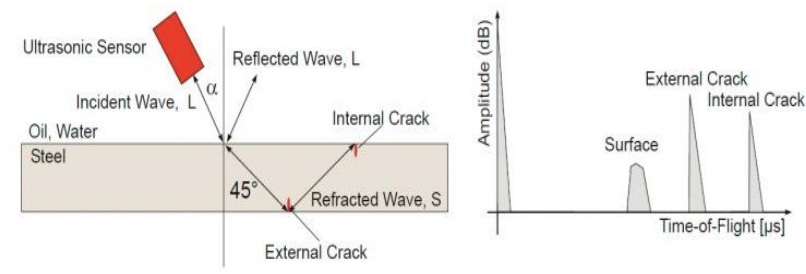

Fig.3. Principle of Ultrasonic measurement

In the common ultrasonic nondestructive testing technology, $45^{\circ}$ wave technique is successfully utilized to achieve the axial crack ultrasonic inspection of pipelines. This technology employs ultrasonic sensors to transmit the ultrasonic waves into pipe wall at a specified incident angle. When ultrasonic waves encounter any reflective characteristics (e.g., edge, crack), some ultrasonic waves are reflected back to the original position. By assessing the time of flight of the echo signal and all kinds of signal characteristics, it can predict the positions and sizes of these characteristics. For a circumferential defect, its length can be determined based on the number of sensors receiving the echo signal. The inspection for circumferential crack defects of girth welds has a different configuration of sensor carrier and data recognition from traditional axial crack inspection. Sensors can be divided into upstream and downstream directions. In addition, each track is equipped with two perpendicular ultrasonic sensors to measure the wall thickness and identify characteristic location. External cracks can be identified by half or one-and-a-half skip reflection, while internal cracks depend on one or two skips for identification. The Principle of sensors in Ultrasonic equipment is shown in FIG.4.

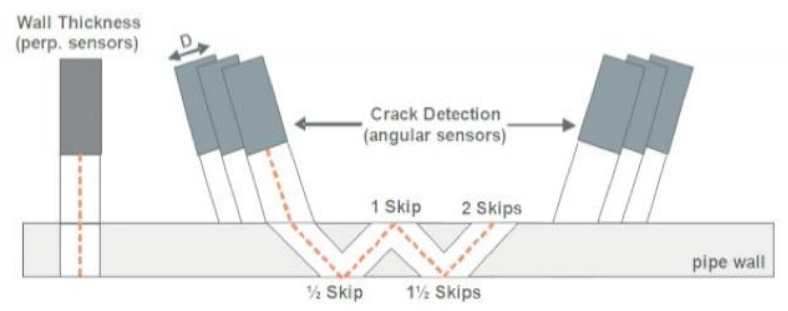

FIG.4. Principle of sensors in ultrasonic equipment

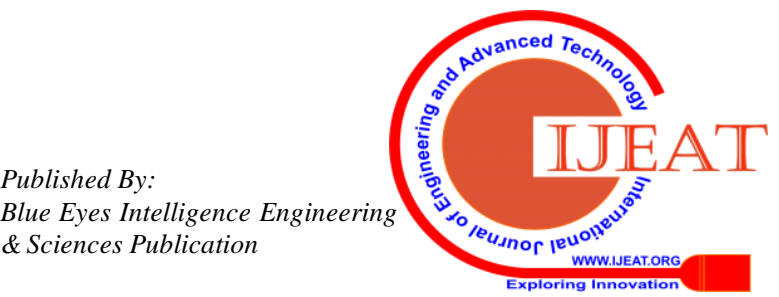




\section{DEFECT DETECTION}

The ultrasonic signals collected by UT tools in pipelines are typically noisy. Effective de-noising techniques are needed to get accurate information regarding defects. This can be done using some signal processing techniques like wavelet transform or Fast Fourier Transform (FFT). Pre-processing of data improves the performance of the neural networks in distinguishing between the defect classes. In the next step, the Artificial Neural Networks (ANNs) are used to distinguish the signals with non-defect (ND) and signals with defects (D) and classify the signal with defect into three types: external corrosion (EC), internal corrosion (IC) and lack of penetration (LP). This essential data can be stored for future reference and planning.

The identification of girth weld defects is analysed on the signals of C-scan view, overlap view and $3-D$ view. In all these views if position of color i.e red and blue in the signal is different from the normal weld characteristics there may be some abnormalities. If there are any abnormalities at the same position in the $\mathrm{C}$ scan view, overlap view and 3-D view the position is recognized as the linear defect characteristics.

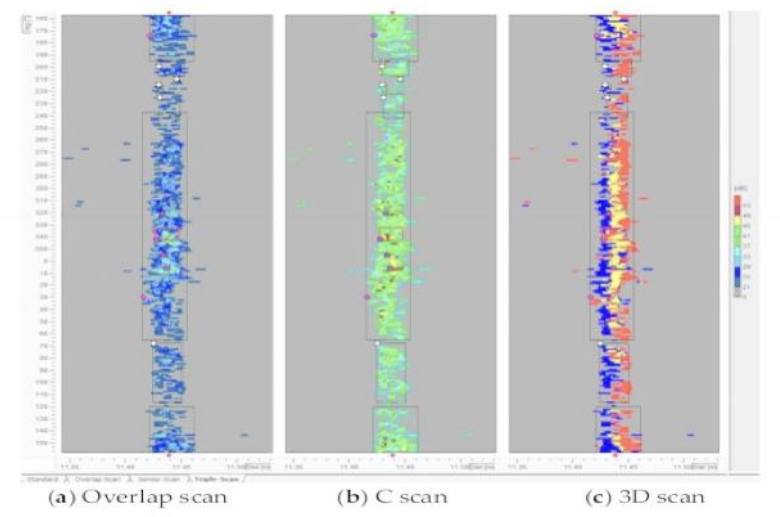

Figure 6. Signal characterisitics of girth weld during ultrasonic testing

Theoretically, the physical measurement of defect depth is conducted based on the number of sampling points. In the experiment process, the zero position of the sensor and the difference between the acoustic velocities of ultrasonic waves in the base metal and weld have a significantisepithe acoustic velocities of ultrasonic waves in the base metal and weld have a significant influence on the accuracy of the experiment. Hence it is necessary to calculate the acoustic velocity accurately.

\section{VI.CONCLUSION}

This paper presents Ultrasonic Testing technique for detecting external and internal defects in a pipeline. Compared with other tools, UT gives reliable defect depth sizing and good repeatability, and it can deal with very small pipeline wall thickness. Compared with MFL tools, UT tools are also sensitive to a larger variety of features. Most defects identified in ultrasonic inspection belonged to geometrical defects, e.g., uneven surface; repair welding, undercut, and slag inclusion, etc. For the ultrasonic in-line inspection of pipelines, the defect identification based on ultrasonic waves is significantly affected by the interior wall of the pipe, air bubbles in motion, and geometry of the girth weld, which should be further studied.

\section{REFERENCES:}

1. Q.Feng,R.Li,B.Nie,S.Liu,L.Zhao,H.Zhang Literature Review:Theory and Application of In- Line inspection Technologies for Oil and Gas Pipeline Girth Weld Defection, Sensors, December 2016. 'istep]

2. Wang, F.; Chen, J. Technical Report on Identification and Evaluation Technology of Magnetic Flux Leakage Detection Signals for Girth Weld Defects; Technical Report QTSXJC-01; PetroChina Pipeline Company: Beijing,bChina, 2015.

3. Ting, W.; Sutherland, J.; Hui, Y. In-Line Inspection and Assessment for Pipeline Girth Weld Defects; PR-469-143508; Pipeline Research Council International: Calgary, AB, Canada, 2015; Volume 4.

4. Zhao, J. Pipe Corrosion Cracking with Ultrasonic Detection Signal Processing Research; Beijing University of Chemical Technology: Beijing, China, 2008

5. .Dai, B.; Zhao, J.; Zhou, Y. Research of Classification and Recognition of Pipeline Corrosion Defectson Ultrasonic In-line Inspection. Mach. Hydraul. 2008, 36, 194-199 [is

6. Yang, Z. Research of Inner Inspection System in the Distance Pipelines; Beijing University of Chemical Technology: Beijing, China, 2007.

7. Chen, G.; Zhang, X.; Xie, C.; He, X. Intelligent Recognition of Crack Depth in Ultrasonic Testing. J. South China Univ. Technol. 2005, 33, 1-5is:

8. Li, Z. Development and Application of Electromagnetic Ultrasonic (EMAT) Technology. Non Destr. Test. 2006, 30, 13-14.

9. Yeomans, M.; Ashworth, B. Development of 36" EmatScan ${ }^{\circledR}$ Crack Detection (CD) Tool. In Proceedings of the 4th International Pipeline Conference, Calgary, AB, Canada, 29 September-3 October 2002; pp. $1543-1548$

10. Song, X.; Huang, S.; Zhao, W. Nondestructive testing technique for cracks in long-distance natural gaspipelines. Nat. Gas Ind. 2006, 26, 103-106

11. Sophian, A.; Tian, G.Y.; Taylor, D.; Rudlin, J. Electromagnetic and eddy current NDT: A review.Insight NonDestr. Test. Cond. Monit. 2001, 3, 1-6.

12. Wu, D.H.; Huang, S.L.; Zhao, W. Transient simulation analysis on magnetic flux leakage detection of cracksin long-distance oil and gas pipeline. Acta Pet. Sin. 2009, 30, 136-140.

13. Wu, D.H.; Huang, S.L.; Zhao, W. Research on 3-D simulation of remote field eddy current detection for pipeline cracks. J. Syst. Simul. 2009, 21, 6626-6629.

14. Willems, H.; Bjorgen, H.P.; Kristiansen, T.-S.; Wileme, G. Qualification of combined ultrasonic inspection tool for detection and sizing of circumferential weld cracks in offshore pipelines. In Proceedings of the 10th International Pipeline Conference, Calgary, AB, Canada, 29 September-3 October 2014.

15. Shan, Q.; Dewhurst, R.J. Surface-breaking fatigue crack detection using laser ultrasound. Appl. Phys. 1993, 62, 14-18. [CrossRef]

16. Levesque, D.; Ochiai, M.; Blouin, A.; Talbot, R.; Fukumoto, A.; Monchalin, J.P. Laser- ultrasonic inspection of surface-breaking tight cracks in metals using SAFT processing. In Proceedings of the IEEE Ultrasonics.Symposium, Prague, Czech Republic, 8-11 October 2002; Volume 1, pp. 753-756.

17. Kandasamy, N., Telagam, N., VR, S. R., \& Arulananth, T. S. (2017). Simulation of Analog Modulation and Demodulation Techniques in Virtual Instrumentation and Remote Lab. International Journal of Online Engineering (iJOE), 13(10), 140-147.

18. J.Surendiran, R.Sridhar, Rajiv arya ," GPRS BASED MONITORING SYSTEM USING IMAGE SENSOR", International Journal of Pure and Applied Mathematics Volume 119 No. 12 2018, 14387-14392 ISSN: 1314-3395.

19. Tse, P.W.; Liu, X.C.; Wang, X.J.; Wang, D. A Novel and Flexible Design of Magnetostrictive Sensorfor Strand/Rope Defect Inspection. In Proceedings of the International Design Engineering TechnicalConferences and Computers and Information in Engineering Conference, Washington, DC, USA,28-31 August 2011.

20. Ye, Y.; Xia, L. Experimental Research on High-Temperature Pipe Corrosion On-Line Monitoring.In Proceedings of the Pressure Vessels and Piping Conference, Toronto, ON, Canada, 15-19 July 2012.

21. C.T.Mgonja The Consequences of cracks formed on the Oil and Gas Pipelines Weld Joints.,IJETT, vol 54 Dec. 17

22. Yorick-Oden-Plants Inspection and Maintenance of crude oil transmission pipelines in the GLSLR region.Sept. 2017 